\title{
Efficient Approach to Characterize Strength Anisotropy in Soils
}

\author{
Zhiwei Gao ${ }^{1}$ and Jidong Zhao ${ }^{2}$
}

\begin{abstract}
Strength anisotropy in soils needs to be characterized by proper anisotropic failure criterion. This paper presents a novel yet simple methodology to generalize an isotropic failure criterion to account for strength anisotropy in soils. A salient ingredient of the method involves the introduction of the degree of cross anisotropy and an anisotropic variable, defined by the joint invariant of the deviatoric stress tensor and the deviatoric fabric tensor, into the frictional characteristic of the isotropic criterion. The well-received Lade's failure criterion is taken as an example to demonstrate the generalization. Predictions using the newly generalized Lade's criterion for a number of soils, including completely decomposed granite, glass beads (virtual sand), natural clays, sand, as well as silty sand, show good agreement with test data. The proposed approach has proved to be simple and generic, and can be effortlessly applied to many existing isotropic failure criteria to adapt them to account for strength anisotropy. The treatment also requires very few parameters, which can be conveniently calibrated from conventional laboratory tests in most cases. DOI: 10.1061/(ASCE)EM.1943-7889.0000451. ( 2012 American Society of Civil Engineers.
\end{abstract}

CE Database subject headings: Anisotropy; Fabrics; Sand (soil type); Clays; Laboratory tests.

Author keywords: Strength anisotropy; Fabric tensor; Joint invariant; Sand; Natural clay; Completely decomposed granite.

\section{Introduction}

Most natural soils are inherently cross-anisotropic because of the depositional processes (Oda and Nakayama 1989; Mitchell and Soga 2005). The isotropic plane frequently coincides with the deposition plane, and its perpendicular direction is often called the axis of anisotropy (Whittle et al. 1994; Abelev and Lade 2004; Callisto and Calabresi 1998). Fabric anisotropy has long been recognized to significantly affect the peak strength of both sands and clays (Casagrande and Carillo 1944). A prominent example is the bearing capacity test of a strip footing on sand examined by Oda et al. (1978) and more recently by Azami et al. (2010), wherein the bearing capacity measured with the loading direction perpendicular to the deposition plane was around 25-34\% higher than that with a loading case parallel to the plane. Similar observations have been found on the strength of clays. For example, Yong and Silvestri (1979) have found that the unconfined compression strength of sensitive St. Louis clay varies continuously with loading directions, with the minimum strength observed being around 60-75\% of the maximum. Strength anisotropy has also been observed in natural/remolded clays by direct shear tests (O'Neill 1985), true triaxial tests (Callisto and Calabresi 1998; Kurukulasuriya et al. 1999; Callisto and Rampello 2002), as well as torsional shear tests (Nishimura et al. 2007; Kumruzzaman and Yin 2010). In practical design of such geostructures as engineered slopes and foundations, the principal stress direction may rotate significantly along a potential slip surface. Proper consideration of strength

${ }^{1} \mathrm{Ph} . \mathrm{D}$. Candidate, Dept. of Civil and Environmental Engineering, Hong Kong Univ. of Science and Technology, Hong Kong SAR, China.

${ }^{2}$ Assistant Professor, Dept. of Civil and Environmental Engineering, Hong Kong Univ. of Science and Technology, Hong Kong SAR, China (corresponding author). E-mail: jzhao@ust.hk

Note. This manuscript was submitted on September 27, 2011; approved on April 9, 2012; published online on April 12, 2012. Discussion period open until May 1, 2013; separate discussions must be submitted for individual papers. This paper is part of the Journal of Engineering Mechanics, Vol. 138, No. 12, December 1, 2012. (CASCE, ISSN 0733-9399/2012/ $12-1447-1456 / \$ 25.00$. anisotropy in these cases becomes necessary for safe assessment of the performance of these structures (Uthayakumar and Vaid 1998). Indeed, Zdravković et al. (2002) have employed a finite-element simulation on a model embankment to demonstrate that more accurate predictions can be achieved if the stress rotation and strength anisotropy of clay are taken into account.

To properly evaluate the anisotropic strength in soil, suitable anisotropic failure criteria need to be developed. In this regard, a variety of approaches have been attempted for different materials. Hill (1950) is among the first to develop an anisotropic yield criterion for general orthotropic materials. By assuming the principal axes of anisotropy to be the reference Cartesian coordinate, Hill (1950) expressed his anisotropic failure criterion in a quadratic form of the Cartesian stress components, which can be reduced to the von Mises criterion when anisotropy is not considered. The criterion however requires proper stress transformation in many cases, which renders it not particularly convenient. A technique by rotating an isotropic yield surface according to the initially anisotropic stress state was later widely adopted to model strength anisotropy of clays (Sekiguchi and Ohta 1977; Anandarajah and Dafalias 1986; Whittle et al. 1994). Because the direction and magnitude of such rotation are typically dependent on the initial stress state, it cannot adequately account for the fabric structure of a soil, for example those features related to particle orientation and void distribution in sand (Kaliakin 2003). To overcome this drawback, attempts have recently been made to characterize the strength of inherently cross-anisotropic soils under true triaxial loading conditions wherein the principal stresses and material fabric are arranged to be coaxial. Abelev and Lade (2004), for example, have developed a failure criterion for cross-anisotropic soils by rotating the isotropic Lade's (1977) failure surface around the origin of the stress space. Another example is the one proposed by Mortara (2010), who introduced a special function of the Lode's angle to expand/shrink the failure curves of an isotropic one in the deviatoric plane. Because these two criteria have been developed for coaxial stress and fabric conditions, the formulations require considerable revision if the material fabric is noncoaxial with the applied stress axes. In addition to the preceding approaches, there is also a class of 
anisotropic failure criteria being developed based on the concept of potential failure plane. Liu and Indraratna (2011), as well as Yao and Kong (2011), for example, have based their developments on the spatial mobilized plane concept (Matsuoka and Nakai 1974) to generalize some existing isotropic criteria to account for anisotropy, by assuming that the peak strength of soils depend on the angle between the spatial mobilized plane and the weak plane of soils (which typically corresponds to the bedding plane). However, these models may become tedious to manipulate in cases of continuous stress rotation, because it would be difficult to determine the angle between the spatial mobilized plane and the weak plane.

More recently, a particularly popular and effective way of formulating an anisotropic failure criterion is through the use of fabric tensor. Soil anisotropy is closely related to the fabric structure formed in a soil. The fabric structure in sand, for instance, may be attributable to the spatial distribution of particles, void space, and contact normal (Oda and Nakayama 1989; Muhunthan et al. 1996; Guo and Stolle 2005). Different forms of fabric tensors have thus been proposed to characterize the internal structure in soils, and have been incorporated in characterizing the constitutive behavior and strength of soils under various loading conditions (Pietruszczak and Mroz 2000, 2001; Li and Dafalias 2002; Azami et al. 2010; Gao et al. 2010). Based on the work by Pietruszczak and Mroz (2000, 2001), Lade $(2007,2008)$ has recently extended his isotropic criterion (Lade 1977) to account for anisotropy by assuming the frictional parameter $\eta$ varies with the loading direction. A similar method has also been followed by Schweiger et al. (2009) based on the MohrCoulomb failure criterion.

The authors propose a general approach to extend an isotropic failure criterion to an anisotropic one in order to characterize the strength anisotropy in soils. This method is based on the use of a fabric tensor as well. Pivotal in this method is the introduction of an anisotropic variable $A$ (Dafalias et al. 2004), defined by the joint invariant of the deviatoric stress tensor and deviatoric fabric tensor, along with the degree of anisotropy, into the frictional descriptor of the isotropic soil criterion. The authors demonstrate that this strategy can be easily applied to any popular isotropic failure criteria for frictional materials, such as the Matsuoka-Nakai failure criterion (Matsuoka and Nakai 1974), the Mohr-Coulomb failure criterion, and the Lade's failure criterion (Lade 1977). The well-received Lade's (1977) isotropic criterion is employed as an illustrative example for generalization using the proposed method. Indeed, a similar previous attempt has been made by the authors on a relatively more complicated failure criterion (Gao et al. 2010). However, by removing the unnecessary procedures and extracting only the essence, the present study aims to put forward a generalpurpose methodology rather than a specific treatment for an individual failure criterion. It will also be shown that with the current approach, an anisotropic failure criterion of elegant form with fewer additional parameters can be easily formulated.

\section{Fabric Tensor and Anisotropic Variable}

A fabric tensor has been frequently used to quantify the anisotropic internal structure formed in soils (Brewer 1964; Oda and Nakayama 1989; Pietruszczak and Mroz 2000, 2001). Fabric anisotropy in granular sands or gravels, for example, is typically governed by the nonspherical particle orientation, contact normal, and void space distributions, whereas for clays, it may represent collectively the preferred orientation of clay platelet and the aggregated/flocculated assemblages. For a soil with cross anisotropy, if the principal axes of the material fabric are aligned with the reference coordinates $(x, y, z)$, with the $x-y$ plane being the isotropic plane, and $z$ the axis of anisotropy, the following fabric tensor $F_{i j}$ has been widely employed to describe the cross anisotropy (Oda and Nakayama 1989)

$$
\begin{aligned}
F_{i j} & =\left[\begin{array}{ccc}
F_{z} & 0 & 0 \\
0 & F_{x} & 0 \\
0 & 0 & F_{y}
\end{array}\right]=\frac{1}{3+\Delta}\left[\begin{array}{ccc}
1-\Delta & 0 & 0 \\
0 & 1+\Delta & 0 \\
0 & 0 & 1+\Delta
\end{array}\right] \\
& =\frac{1}{3}\left[\begin{array}{lll}
1 & 0 & 0 \\
0 & 1 & 0 \\
0 & 0 & 1
\end{array}\right]+\frac{2 \Delta}{9+3 \Delta}\left[\begin{array}{ccc}
-2 & 0 & 0 \\
0 & 1 & 0 \\
0 & 0 & 1
\end{array}\right]
\end{aligned}
$$

$\Delta$ in Eq. (1) was originally employed by Oda and Nakayama (1989) as a measure of anisotropy distribution of nonspherical particles in granular sand. It indeed in a wider sense can be used to denote the degree of fabric anisotropy for a general class of geomaterials. including clays and rocks as well. Its value typically varies from 0 when the fabric is isotropic, to unity when the degree of anisotropy is the maximum. For most soils, $0<\Delta<1$ is the typical range. The fabric tensor expressed in Eq. (1) may be subjected to orthogonal transformation if the reference frame changes or the material fabric plane is rotated.

As previously mentioned, the strength of soil depends on how the loading is applied relative to the bedding plane of the soil. To predict the behavior of strength anisotropy, it is therefore important to properly quantify the relative orientation between the stress direction and material fabric in the three-dimensional space. According to representation theory of tensors (Wang 1970), the relative orientation between the (second-order) fabric tensor and the (second-order) stress tensor can be represented by the following four joint invariants between the deviatoric part of the fabric tensor and the deviatoric stress tensors, as follows:

$$
\begin{aligned}
& A=\frac{s_{i j} d_{i j}}{\sqrt{s_{m n} s_{m n}} \sqrt{d_{m n} d_{m n}}} \\
& B=\frac{s_{i j} d_{j k} d_{k i}}{\sqrt{s_{m n} s_{m n}}\left(\sqrt{d_{m n} d_{m n}}\right)^{2}} \\
& C=\frac{s_{i j} s_{j k} d_{k i}}{\left(\sqrt{s_{m n} s_{m n}}\right)^{2} \sqrt{d_{m n} d_{m n}}} \\
& D=\frac{s_{i j} s_{j k} d_{k l} d_{l i}}{\left(\sqrt{s_{m n} s_{m n}}\right)^{2}\left(\sqrt{d_{m n} d_{m n}}\right)^{2}}
\end{aligned}
$$

where $s_{i j}\left(=\sigma_{i j}-p \delta_{i j}\right)$ and $d_{i j}\left(=F_{i j}-F_{k k} \delta_{i j} / 3\right)$ are the deviatoric parts of the stress tensor $\sigma_{i j}$ and fabric tensor $F_{i j}$, respectively; and $p\left(=\sigma_{k k} / 3\right)$ is the mean stress and $\delta_{i j}(=1$ for $i=j$ and $=0$ for $i \neq j)$ is the Kronecker delta.

A torsional shear test on cross anisotropic soil is taken as an example to illustrate the feature of the four joint invariants. If the soil is cross anisotropic and can be characterized by a fabric tensor in form of Eq. (1), the variations of the four normalized joint invariants with the angle between the major principal stress direction with respect to the bedding axis (vertical), $\alpha$, and the intermediate principal stress ratio $b\left[=\left(\sigma_{2}-\sigma_{3}\right) /\left(\sigma_{1}-\sigma_{3}\right)\right]$, are shown in Figs. 1(b-e). Here, $\sigma_{1}, \sigma_{2}$, and $\sigma_{3}$ are the major, intermediate, and minor principal stress, respectively. As for the expression for the material fabric $\left(d_{i j}\right)$ and applied stress condition $\left(s_{i j}\right)$ under such loading conditions, please refer to the Appendix. From Fig. 1, A increases monotonically with $\alpha$ at constant $b$ values, while decreases 
(a)

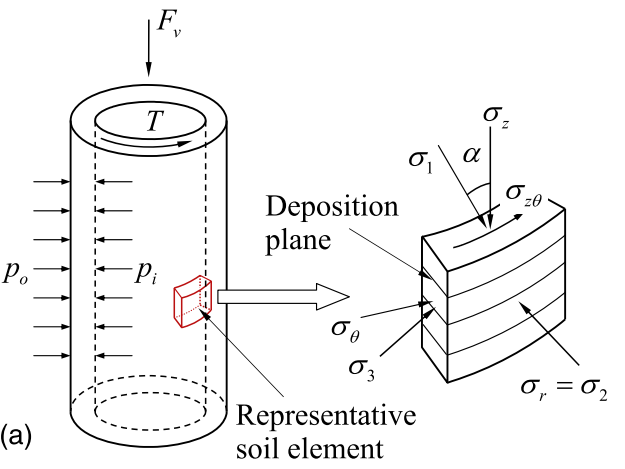

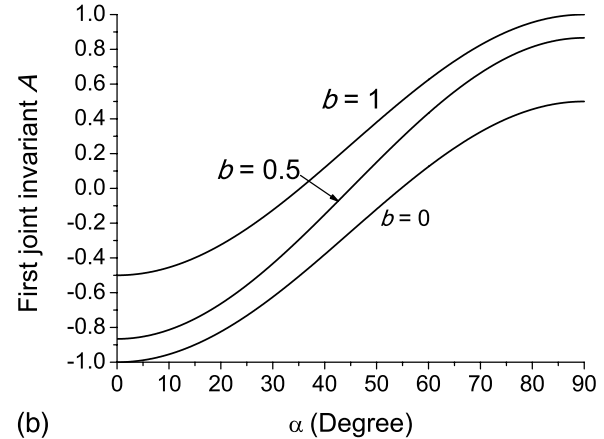

(b)
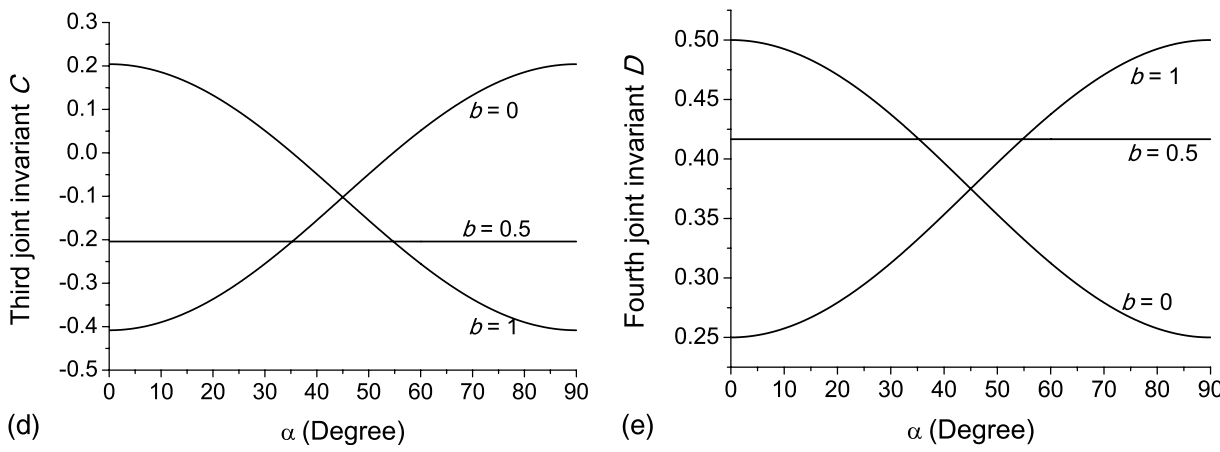

Fig. 1. (a) Stress state in torsional shear tests (modified from Yoshimine et al. 1998); (b)-(e) variations of the four joint invariants with the change of $\alpha$ at different constant $b$ values

with $b$ at the same $\alpha$. $B$ varies in an opposite trend of $A$. Also, $C$ and $D$ do not vary with $\alpha$ and $b$ in a similar way as $A$ and $B$, for example, they are not simply monotonically. For example, $C$ decreases with $\alpha$ at $b=0$, while increases with $\alpha$ at $b=1$. The monotonic feature of $A$ or $B$ makes them easy to manipulate and suitable to be used for constitutive characterization of soils. Indeed, the first joint invariant $A$, in its relative simpler form as compared with the other three, has been employed to characterize the strength anisotropy of geomaterials (Gao et al. 2010) and to describe the effect of inherent anisotropy on sand behavior as well (Dafalias et al. 2004). Comparatively, neither $C$ nor $D$ can be employed to describe the soil anisotropy independently. For instance, $C$ or $D$ is a constant at $b=0.5$, which renders them inappropriate to describe the anisotropic behavior in a soil, unless they are used in conjunction with $A$ and $B$.

In addition to the degree of anisotropy $\Delta$, the first joint invariant $A$, which is also termed as the anisotropic variable, will be employed in formulating the anisotropic failure criterion for soils. To facilitate the criterion description and parameter calibration, the expression for $A$ under some typical loading conditions (torsional shear and true triaxial tests) will be given (a detailed calibration procedure is shown in the Appendix). Under a typical torsional shear test condition, as shown in Fig. 1(a), the expression for $A$ is

$$
A=\frac{-3 \cos ^{2} \alpha+(b+1)}{2 \sqrt{b^{2}-b+1}}
$$

Furthermore, when the pressures inside and outside the cylinder are kept the same in the torsional shear apparatus $\left[p_{i}=p_{o}\right.$ in Fig. 1(a)], we get $b=\sin ^{2} \alpha$ (Lade et al. 2008), and Eq. (6) can be further simplified as

$$
A=\frac{2 b-1}{\sqrt{b^{2}-b+1}}
$$

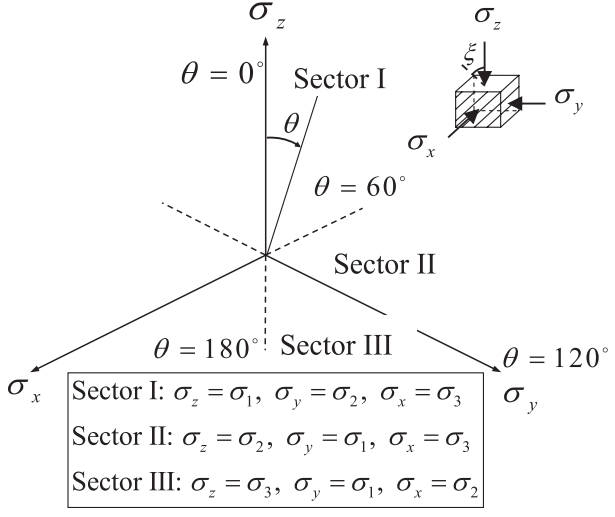

Fig. 2. Illustration of true triaxial tests on cross-anisotropic soils (from Ochiai and Lade 1983)

In typical true triaxial tests, as shown in Fig. 2, $A$ can be collectively expressed as follows:

$$
A=\left\{\begin{array}{cl}
\frac{3(b-1) \cos ^{2} \xi+(1-2 b)}{2 \sqrt{b^{2}-b+1}} & \text { Sector I }\left(0 \leq \theta \leq 60^{\circ}\right) \\
\frac{3(1-b) \cos ^{2} \xi+(b-2)}{2 \sqrt{b^{2}-b+1}} & \text { Sector II }\left(60 \leq \theta \leq 120^{\circ}\right) \\
\frac{3 \cos ^{2} \xi+(b-2)}{2 \sqrt{b^{2}-b+1}} & \text { Sector III }\left(120 \leq \theta \leq 180^{\circ}\right)
\end{array}\right.
$$

where $\xi=$ angle between the vertical direction and the material axis of anisotropy. For a detailed discussion on the variation of $A$, please 
refer to Gao et al. (2010). In addition to $A$, strength anisotropy of a soil is apparently dependent on the degree of fabric anisotropy characterized by $\Delta$ as well. Both $\Delta$ and $A$ will hence be used to formulate the subsequent anisotropic failure criterion for soils.

\section{General Approach to Characterize Strength Anisotropy}

\section{Generalization of Isotropic Failure Criterion to Account for Cross Anisotropy}

It is assumed that the failure criterion for an isotropic material (e.g., the Mohr-Coulomb failure criterion, the Drucker-Prager failure criterion, the Matsuoka-Nakai failure criterion, and Lade's failure criterion) can be expressed in the following functional form:

$$
f_{I}=\hat{f}_{I}\left(\sigma_{i j}, \chi_{r}\right)
$$

where $f_{I}=$ general expression of isotropic failure criterion for clay, sand, or rock, and $\chi_{r}=$ set of relevant strength parameters (e.g., cohesion and internal friction angle in the Mohr-Coulomb failure criterion). Our approach to generalize it to a cross anisotropic one is straightforward. As previously mentioned, the influence of material fabric anisotropy on strength may be reflected by two variables, $A$ and $\Delta$, and both can be considered through their effects on the model parameters, for example

$$
f_{A}=\hat{f}_{A}\left(\sigma_{i j}, \chi_{n}\right)=\hat{f}_{A}\left[\sigma_{i j}, \hat{\chi}_{r}(A, \Delta)\right]
$$

where $f_{A}=$ general expression of anisotropic failure criteria for soils. $\chi_{n}=\hat{\chi}_{r}(A, \Delta)$, and $\hat{\chi}_{r}$ denotes a functional form of $\chi_{r}$ involving $A$ and $\Delta$ (Pietruszczak and Mroz 2000). While any proper functional form of $\hat{\chi}_{r}$ may serve a good purpose for the generalization, the following specific expression for $\chi_{n}$ is proposed:

$$
\chi_{n}=\chi_{r} \exp [g(A, \Delta)]
$$

where $\chi_{r}$ takes the original form in the base isotropic failure criterion, and $g(A)$ is an anisotropic (interpolation) function of the following general form (Pietruszczak and Mroz 2000, 2001):

$$
g(A, \Delta)=\Delta\left[c_{1}(1+A)+c_{2}(1+A)^{2}+\ldots c_{n}(1+A)^{n}\right]
$$

where $c_{i}(i=1, \ldots, n)$ denote a set of material parameters. The following observations can be made from Eqs. (11) and (12):

1. If the soil is isotropic, we have $\Delta=0, g(A, \Delta) \equiv 0$, and $\chi_{n} \equiv \chi_{r}$ [Eqs. (11) and (12)]. The anisotropic failure criterion will readily recover the reference isotropic one as a special case.

2. For cross-anisotropic soils with $0<\Delta \leq 1$, with nonzero $c_{i}(i=1, \ldots, n), g(A, \Delta)$ generally varies with the loading direction characterized by $A$, as well as degree of anisotropy characterized by $\Delta$. In the conventional triaxial compression shear mode with the major principal stress direction being perpendicular to the isotropic plane (e.g., the shear mode corresponding to $\alpha=0^{\circ}$ at $b=0$ in Fig. 1(a) or $\theta=0^{\circ}$ with $\xi=0^{\circ}$ in Fig. 2), one always has $A=-1$ [Eqs. (6) and (8)], $g(A, \Delta) \equiv 0$, and $\chi_{n} \equiv \chi_{r}$ [Eqs. (11) and (12)]. This implies that the expression for the anisotropic failure criterion is identical to that of the reference isotropic one under such special loading conditions, and the corresponding test results can be used to determine $\chi_{r}$. In other shear modes with
$A \neq-1$, the values of $\chi_{n}$ would generally be different from $\chi_{r}$ for cross-anisotropic soils, and the test results can be employed to calibrate $c_{i}(i=1, \ldots, n)$.

3. In most applications, the orientation of the isotropic planes is easily known a priori ( $A$ can be calibrated), whereas the degree of fabric anisotropy $\Delta$ may not be conveniently measured. In dealing with this difficulty, one may treat the combined terms $c_{i} \Delta$ as a single material parameter $\bar{c}_{i}=c_{i} \Delta$, and calibrate $\bar{c}_{i}$ instead of determining the exact value of $\Delta$. This method will be followed in the subsequent sections.

\section{Generalization of Lade's (1977) Failure Criterion}

As an illustrative example, the well-received isotropic failure criterion proposed by Lade (1977) will be extended using the previously mentioned approach to characterize strength anisotropy. As evidenced by many experimental investigations, fabric anisotropy mainly affects the frictional characteristics of soils (Lade 2008; Kumruzzaman 2008; Kumruzzaman and Yin 2010). Following the generalization approach outlined in last section, the following generalized Lade's failure criterion is proposed:

$$
f=\left(\frac{\bar{I}_{1}^{3}}{\bar{I}_{3}}-27\right)\left(\frac{\bar{I}_{1}}{p_{a}}\right)^{m}=\eta=\eta_{r} \exp [g(A, \Delta)]
$$

where $m=$ nonnegative parameter of Lade's (1977) isotropic criterion controlling the curvature of the failure curve in the meridian plane. $p_{a}$ is the atmospheric pressure $(101 \mathrm{kPa}) ; \eta_{r}$ is a parameter characterizing the frictional property of soils; $\bar{I}_{1}\left(=\bar{\sigma}_{1}+\right.$ $\left.\bar{\sigma}_{2}+\bar{\sigma}_{3}\right), \bar{I}_{3}\left(=\bar{\sigma}_{1} \bar{\sigma}_{2} \bar{\sigma}_{3}\right)$ are the first and third invariants of the transformed stress tensor $\bar{\sigma}_{i j}$ (Lade 1997) expressed as

$$
\bar{\sigma}_{i j}=\sigma_{i j}+\sigma_{0} \delta_{i j}
$$

where $\sigma_{0}=$ triaxial tensile strength of a soil. While the anisotropic function in form of Eq. (12) is general, it is normally sufficient to consider only the first term for most soils, for example, $g(A, \Delta)=$ $c_{1} \Delta(1+A)$. Nevertheless, for some natural clays, the minimum peak strength is not observable when the major principal stress direction is parallel to the deposition plane at the same $b$ value (Nishimura et al. 2007). In this case, including the $c_{2}$ term may lead to better predictions. Depending on the applied soil type, the following two forms of anisotropic interpolation function for Eq. (13) are employed:

$$
\begin{gathered}
g(A, \Delta)=c_{1} \Delta(1+A)=\bar{c}_{1}(1+A) \\
g(A, \Delta)=\Delta\left[c_{1}(1+A)+c_{2}(1+A)^{2}\right] \\
=\bar{c}_{1}(1+A)+\bar{c}_{2}(1+A)^{2}
\end{gathered}
$$

\section{Calibration of the Failure Criterion}

An essential step to make the proposed failure criterion useful is the calibration of relevant parameters. A general principle for model calibration is to use easily performed (or accessible) tests (either in the laboratory or in the field) as much as possible, such as conventional triaxial tests or simple shear tests. To this end, the completely decomposed granite in Hong Kong (HK-CDG) is employed as an illustrative example. The HK-CDG has been investigated extensively by Kumruzzaman (2008) through conventional triaxial tests, plane strain tests, true triaxial tests, as well as torsional shear tests. Though these tests cover the whole range of $b$ and $\alpha$ (or $\xi$ ), the triaxial compression and extension tests will be used to calibrate 
a failure criterion in the form of Eq. (13), while leaving other test data for the purpose of verification. The following summarize the calibration procedures:

1. Determination of the triaxial tensile strength parameter $\sigma_{0}$. For reconstituted soils, such as the HK-CDG, $\sigma_{0}$ can be set to 0 , because the interparticle cohesion is normally negligible. As for natural clays and cemented soils, there may be interparticle adhesion because of chemical bonding formed by cementation of carbonates, silica alumina, iron oxide, and organic compounds (Mitchell and Soga 2005). In this case, $\sigma_{0}>0$. Reliable value of $\sigma_{0}$ should be obtained by triaxial testing data at relatively low effective stresses.

2. Determination of $m$ and $\eta_{r}$ in Eq. (13). They both can be calibrated based on test data from conventional triaxial compression tests by plotting $\ln \left(p_{a} / \bar{I}_{1}\right)$ versus $\ln \left(\bar{I}_{1}^{3} / \bar{I}_{3}-27\right)$ at failure and locating the best fit straight line. The intersection of this fitting line with the vertical axis at $\ln \left(p_{a} / \bar{I}_{1}\right)=0$. $\left(p_{a} / \bar{I}_{1}=1\right)$ is $\ln \left(\eta_{r}\right)$ and its slope is $m$ (Lade 1997, 2008). Fig. 3(a) illustrates the calibration of $m=0$ and $\eta_{r}=23.8$ for HK-CDG according to the solid regression line. Actual prediction of our failure criterion in triaxial compression is compared against the original test data in Fig. 3(b). In the shear model of triaxial compression, $A=-1, g(A, \Delta)=0$, such that the predictions of the isotropic and anisotropic criteria are identical [Eqs. (13) and (15a)].

3. Calibration of parameters $\bar{c}_{i}(i=1, \ldots, n)$. They can be calibrated based on the value of $\eta$ in other shear modes where $A \neq-1$. In most cases, such as for HK-CDG, only the first term is sufficient and the anisotropic interpolation function in Eq. (15a) is adopted. For HK-CDG, according to the dash regression line in Fig. 3(b) for triaxial extension obtained by using the same $m$ value previously determined [the variation of $m$ with loading direction is neglected here as shown in Eq. (13)], one can get $\eta_{e}=\exp (2.43)=11.36$ [Fig. 3(a)]. Because $A=A_{e}=1$ in this shear mode $\left[\alpha=90^{\circ}\right.$ at $b=1$ in Fig. 1(a)], one has $\eta_{e}=\eta_{r} \exp \left(2 c_{1} \Delta\right)=\eta_{r} \exp \left(2 \bar{c}_{1}\right)$ [Eqs. (13) and (15a)]. Hence, $\bar{c}_{1}=c_{1} \Delta=-0.37$ can be obtained based on the predetermined value for $\eta_{r}$.

\section{Verification of the Anisotropic Failure Criterion}

The proposed anisotropic failure criterion in Eq. (13) will be further verified by a wide range of different soils. In addition to HK-CDG, the authors shall also employ test results on glass beads (virtual sand), natural clays (Pietrafitta clay and Pisa clay), Toyoura sand, as well as silty sand for this purpose.

\section{Hong Kong Completely Decomposed Granite}

Fig. 3 shows the predictions of the isotropic and anisotropic criteria for the failure of HK-CDG. The predictions of the Lade's (1997) isotropic criterion are obtained by setting $g(A, \Delta)=0$ and $\eta=\eta_{r}$, according to Eq. (13). While relevant parameters have been calibrated by conventional triaxial testing data, as previously shown, the data obtained from torsional shear tests and true triaxial tests will be employed for verification of this soil. The torsional shear tests on HK-CDG by Kumruzzaman (2008) cover the whole range of $b$ and $\alpha$, while the true triaxial tests are conducted only in Sector I with constant mean stress under drained conditions (see Fig. 2 for the sector partition). As shown in Fig. 3, the anisotropic criterion offers overall satisfactory predictions, but appears to overestimate the tested friction angle $\varphi\left(=\operatorname{arc} \sin \left[\left(\sigma_{1}-\sigma_{3}\right) /\left(\sigma_{1}+\sigma_{3}\right)\right]\right)$ at around $b=0.5$ and $\alpha=45^{\circ}$ [Fig. 3(c)], $b=0.5$ and $\alpha=2^{\circ}$ [Fig. 3(d)], and $b=0.06$ and $\alpha=45^{\circ}$ [Fig. 3(e)]. The maximum overestimation is no greater than $10 \%$. In contrast, the isotropic criterion appears to overestimate the strength of the soil in most cases, as compared with the test results. It nevertheless gives slightly better prediction for the true triaxial test data in Sector I [Fig. 3(f) and (g)] than the anisotropic criterion. Indeed, as remarked by Abelev and Lade (2004), the anisotropic effect in this range of Section I is not significant, and an isotropic criterion may suffice for strength characterization on most occasions.

\section{Glass Beads (virtual sand)}

Haruyama (1981) has conducted a group of true triaxial tests on spherical glass beads, of which the peak strength has been found to be strongly anisotropic. The test setup is identical to that shown in Fig. 2, with $\xi=0^{\circ}$ and the mean stress $p$ kept constant at $294 \mathrm{kPa}$ in all the tests. Because there is no interparticle cohesion for this material, $\sigma_{0}=0$ is adopted. The parameter $m$ was also set to 0 , because there was no sufficient data to calibrate the curvature of the failure curve in the meridian plane. $\eta_{r}=12.9$ is determined according to the friction angle $\varphi_{c}=28.6^{\circ}$ at $\theta=0^{\circ}$ [Fig. 4(b)]. Meanwhile, at $\theta=180^{\circ}, \eta_{e}=7.79$, and $A_{e}=1$, it is readily obtained that $\bar{c}_{1}=c_{1} \Delta=-0.25$, based on the anisotropic function in Eq. (15a).

With these parameters, the criterion predictions are comparatively presented in Fig. 4 with the test data. The anisotropic criterion gives reasonable predictions on the shear strength of the glass bead in the deviatoric plane, whereas the isotropic criterion generally overpredicts the material strength in the range of $90 \leq \theta \leq 180^{\circ}$ [Fig. 4(a)]. Fig. 4(b) shows the predicted variation of friction angles in the three sectors. The isotropic criterion predicts one single $b-\varphi$ relationship, which is obviously inconsistent with the tests data, while the anisotropic criterion gives three different $b-\varphi$ relationships, which are close to the test data in the three sectors. Notably, however, at $b=0.25$ and $b=0.5$ in Sector II as well as $b=0.25$ in Sector III, the prediction by the anisotropic criterion is about $7 \%$ higher than the measured one [Fig. 4(b)]. In the deviatoric plane, the distance of the stress point from the center is set to $\sqrt{2 / 3} q$, where $\left(q=\sqrt{3 s_{i j} s_{i j} / 2}\right)$ is the deviatoric stress.

\section{Natural Pietrafitta Clay}

A series of conventional triaxial compression tests and true triaxial tests were performed by Callisto and Rampello (2002) on natural Pietrafitta clay to investigate its strength anisotropy. All the true triaxial tests were conducted at the same mean stress $p=250 \mathrm{kPa}$ under drained conditions. Because no test data of triaxial tests at very low confining pressure are available for Pietrafitta clay, it is not possible to calibrate reliable value for its triaxial cohesion. The authors hereby assume $\sigma_{0}=0$. The two parameters, $\eta_{r}=34.7$ and $m=0.68$, are calibrated based on the regressed straight line shown in Fig. 5(a), by following the same procedure previously discussed for HK-CDG. The parameter $\bar{c}_{1}=c_{1} \Delta=0.4$ is determined based on the test data at $\theta=180^{\circ}\left(\eta_{e}=76.6, A=1\right)$ and Eq. (15a). The corresponding criterion predictions are shown in Figs. 5(b and c) in comparison with the test data. Notable underestimations can be observed by the predictions compared with the test data in Section I in the deviatoric plane. This might be caused by the fact that $\eta_{r}$ is determined according to the whole test data in conventional triaxial compression [Fig. 5(b)].

\section{Natural Pisa Clay}

Stress path-controlled triaxial and true triaxial tests have been carried out by Callisto and Calabresi (1998) on natural soft clay, Pisa clay. The soil tested was sampled from the upper clayey deposit 

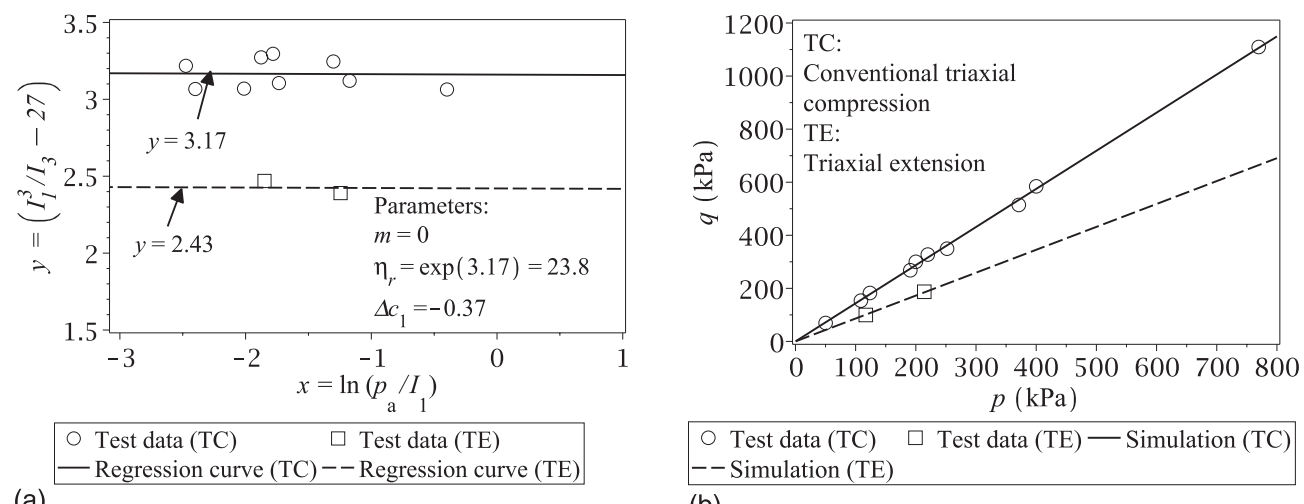

(b)
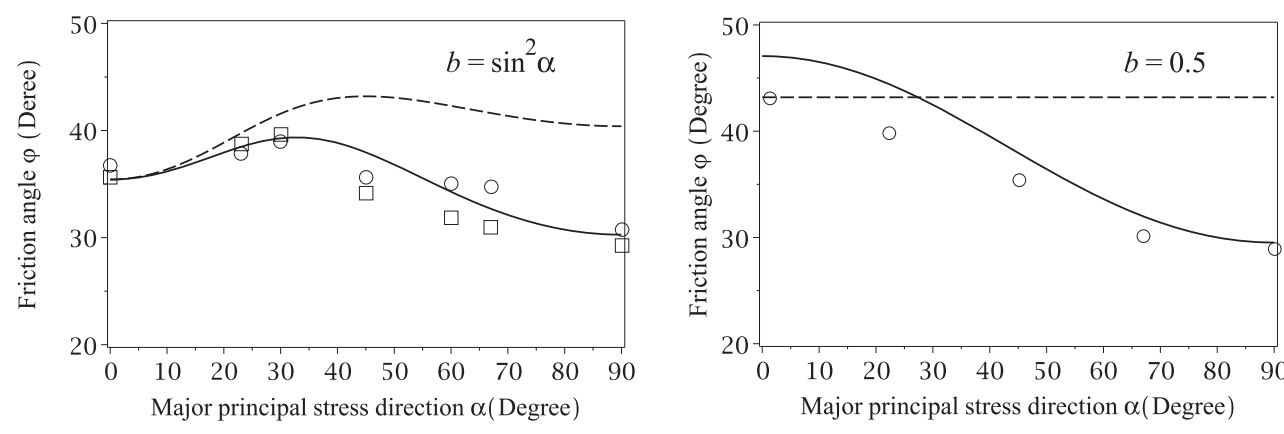

(c)
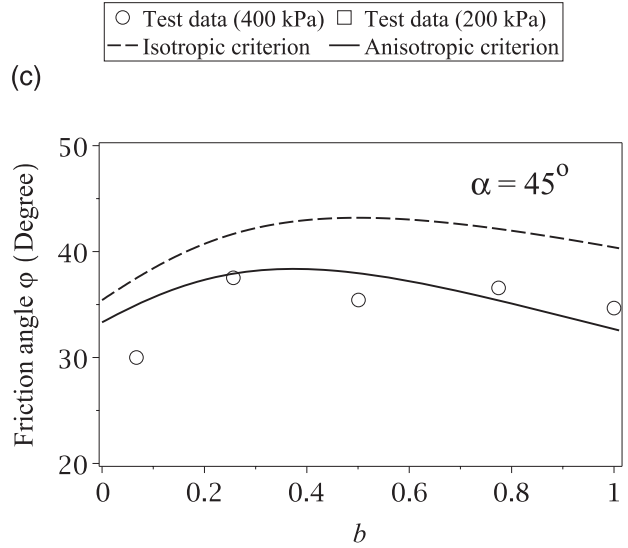

(d)
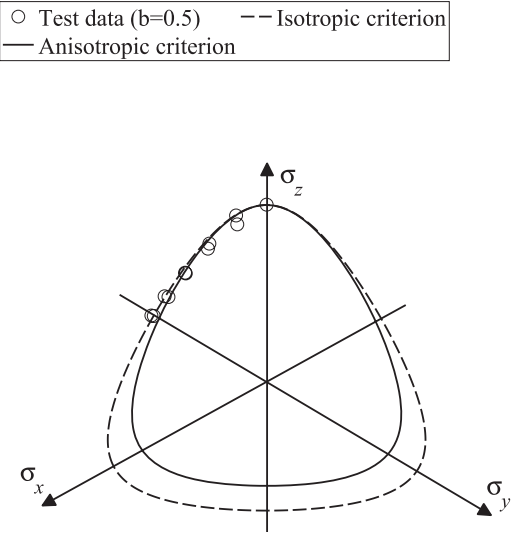

True triaxial test data-- Isotropic criterion
- Anisotropic criterion

(f)

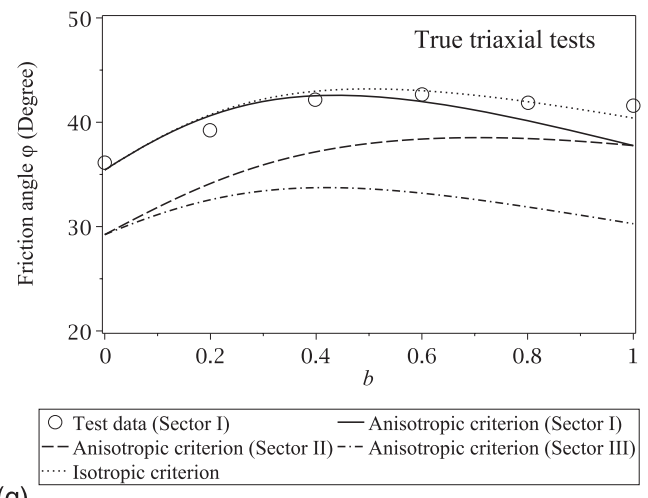

(g)

Fig. 3. (a) Illustration on calibrating the parameters $m, \eta_{r}$, and $c_{1} \Delta\left(=\bar{c}_{1}\right)$ for Hong Kong completely decomposed granite; (b) comparison between the anisotropic criterion simulation and test results in triaxial compression and extension, and comparison of the predictions of the anisotropic and the isotropic criteria for failure of Hong Kong completely decomposed granite with data in torsional shear tests with (c) $b=\sin ^{2} \alpha$, (d) $b=0.5$, and (e) $\alpha=45^{\circ}$, as well as in (f) and (g) true triaxial tests [test data from Kumruzzaman (2008)] 


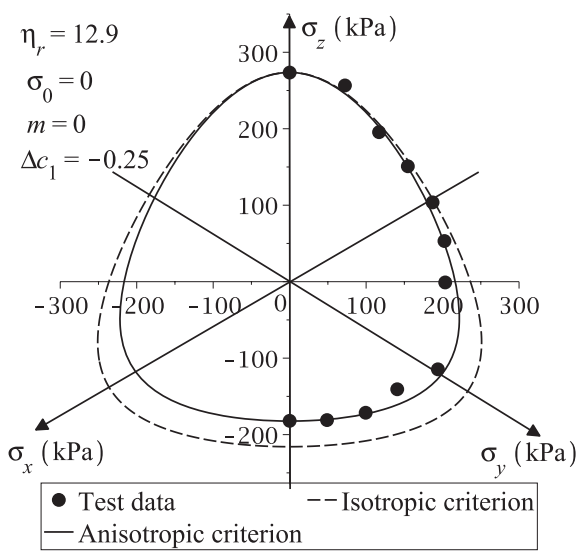

(a)

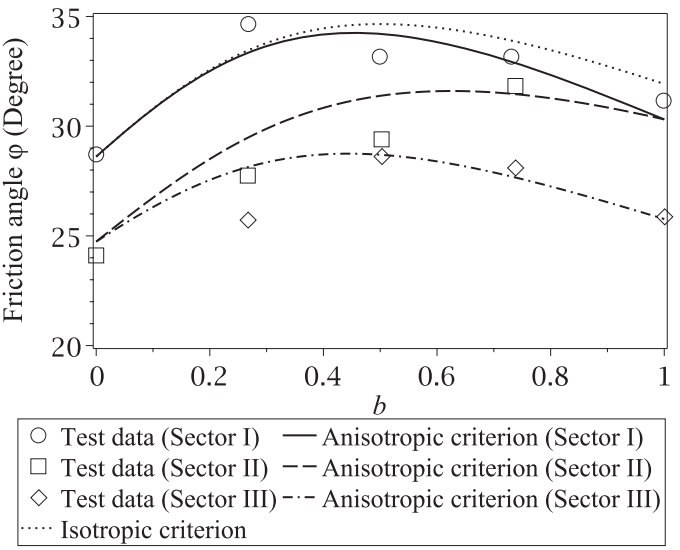

(b)

Fig. 4. Verifications of the isotropic and anisotropic criteria for test results on glass beads in (a) the deviatoric plane; (b) the $b-\varphi$ plane [test data from Haruyama (1981)]

found below the Tower of Pisa. All the samples were reconsolidated to the in situ stress state and were then sheared to failure with a constant mean stress of $88.2 \mathrm{kPa}$ under drained conditions. Because there are no sufficient data to determine the curvature of the failure curves in the meridian plane as well as the triaxial tensile strength, $m=0$ and $\sigma_{0}=0$ are assumed. $\eta_{r}=18.2$ is determined according to the failure stress at $\theta=0$ (Fig. 6). The two-term form anisotropic function in Eq. (15b) is employed to describe the strength variation. In particular, two shear modes, $S_{1}$ and $S_{2}$, as shown in Fig. 6, are employed to determine $c_{1} \Delta$ (or $\bar{c}_{1}$ ) and $c_{2} \Delta$ (or $\left.\bar{c}_{2}\right)$. At shear mode $S_{1}, \eta_{1}=19.05$ and $A_{1}=0.2$, and at shear mode $S_{2}$, $\eta_{2}=14.56$ and $A_{2}=0.75$. In conjunction with Eqs. (13) and (15b), we obtain $\bar{c}_{1}=c_{1} \Delta=0.4$ and $\bar{c}_{2}=c_{2} \Delta=-0.3$, respectively. The same procedure may be followed to calibrate an expression of the anisotropic function with more terms to get more accurate results (Pietruszczak and Mroz 2000, 2001; Azami et al. 2010). Fig. 6 indicates that the anisotropic criterion gives better predictions than the isotropic one, despite certain underestimations around $\theta=60^{\circ}$.

\section{Toyoura Sand}

A series of true triaxial tests and plane strain tests were carried out on Toyoura sand by Lam and Tatsuoka (1988) and Tatsuoka et al. (1990) to investigate its strength anisotropy. Relevant strength parameters in the form of Eq. (13) for Toyoura sand are determined as follows. First, $\eta_{r}=38.7$ is calculated according to the friction angle $\varphi_{c}=41^{\circ}$ at $\theta=0^{\circ}$ by setting $\sigma_{0}=m=0$. By using the one-
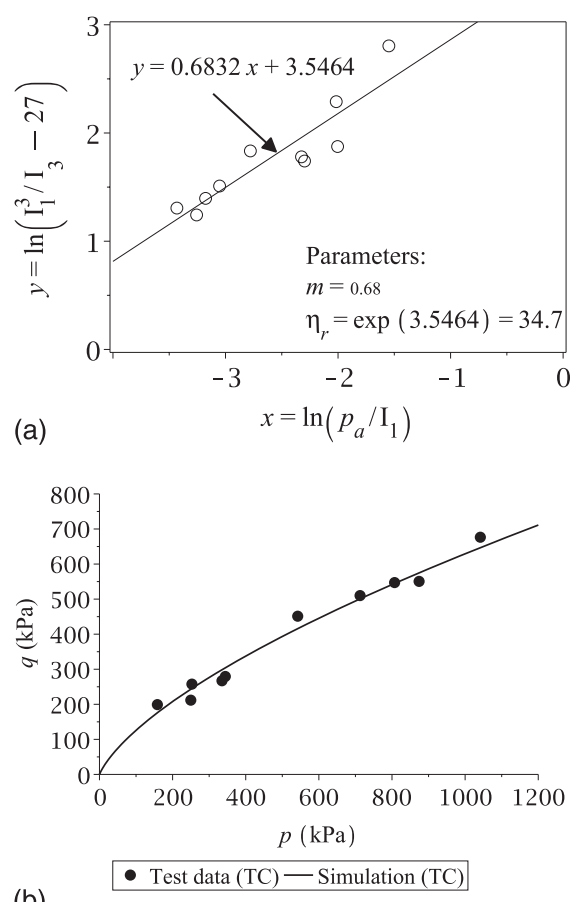

(b)

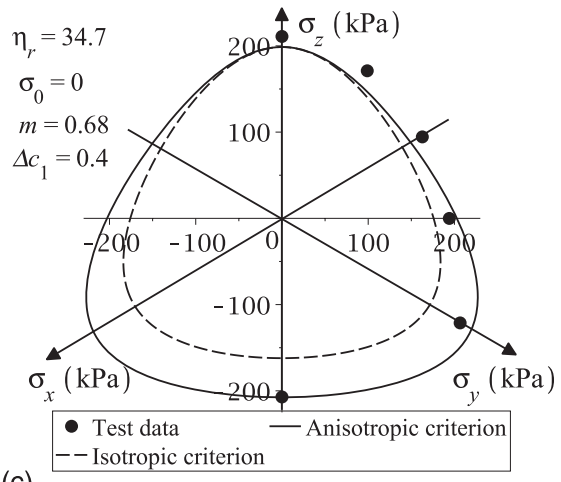

(c)

Fig. 5. Verification of the anisotropic failure criterion for test data on natural Pietrafitta clay [test data from Callisto and Rampello (2002)]: (a) calibration of parameters $\eta_{r}$ and $m$; (b) comparison of test results in conventional triaxial compression with the criterion predictions; (c) comparison between the true triaxial test results and predictions by the isotropic and anisotropic criteria

term expression in Eq. $(15 a), \bar{c}_{1}=c_{1} \Delta=-0.2$ is then calibrated based on the stress state at $\theta=180^{\circ}$ following the same procedure previously discussed for HK-CDG. Because the measured $b$ value in the plane strain tests is between 0.2 and 0.3 (Tatsuoka et al. 1990), the predictions by the isotropic and anisotropic criteria are obtained by considering these two $b$ values [Fig. 7(c)]. Because the intermediate principal stress $\sigma_{2}$ is always along the zero-strain direction, the stress state in plane strain tests is similar to that in torsional shear tests, as shown in Fig. 1(a). Therefore, the expression for $A$ can be calculated using Eq. (6). The predictions by the failure criteria are presented in Fig. 7. Evidently, the anisotropic criterion can capture the strength variation of Toyoura sand in true triaxial tests [Fig. 7(a)] and triaxial compression tests [Fig. 7(b)] satisfactorily, but overestimates the friction angle in plane strain tests [Fig. 7(c)]. The following reasons may account for the observed discrepancies. First, the predicted friction angle by both the isotropic and anisotropic criteria is higher than the measured value at $\alpha=0^{\circ}$. This test condition corresponds to a shear mode between $\theta=11^{\circ}(b=0.2)$ 


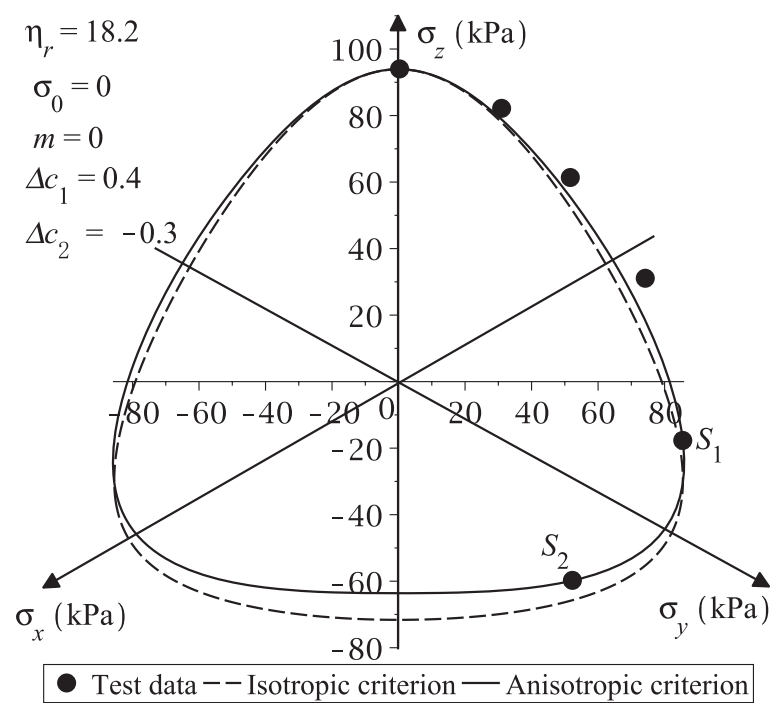

Fig. 6. Characterization of the failure of Pisa clay by the isotropic and anisotropic criteria compared with the test data [test data from Callisto and Calabresi (1998)]; two-term equation in Eq. (15b) is used for the anisotropic interpolation function

and $\theta=17^{\circ}(b=0.3)$, shown in Fig. 7(a), and both of the isotropic and the anisotropic criteria overestimate the tested peak strength in this range from the true triaxial tests. Second, the minimum friction angle is observed around $\alpha=60-70^{\circ}$, but the anisotropic criterion gives a minimum value at $\alpha=90^{\circ}$. This may be attributable to different formations of shear band in true triaxial tests and in plane strain tests (Lam and Tatsuoka 1988; Tatsuoka et al. 1990). In addition to the fabric anisotropy, the strength of the soil may also be affected by shear band formation, as observed by Lade et al. (2008).

\section{Nevada II/ATC Silty Sand}

Shapiro and Yamamuro (2003) performed a group of true triaxial tests on Nevada II sand with $20 \%$ ATC silt by weight. The strength of this sand has been found to be anisotropic. In calibrating the related parameters, $m=0.071$ and $\eta_{r}=22.9$ are directly obtained from the data provided by Shapiro and Yamamuro (2003), and $\sigma_{0}=0$ is adopted, because the material is cohesionless. Eq. (15a) is used for the anisotropic interpolation function, and $\bar{c}_{1}=c_{1} \Delta=-0.32$ is calculated according to the failure stress state in conventional triaxial extension with $\theta=180^{\circ}\left(\eta_{e}=12.1\right.$ and $\left.A_{e}=1\right)$ in Fig. 8(a). As seen from the predicted results in Fig. 8, the anisotropic criterion can capture the strength anisotropy of this sand reasonably well, but somehow overestimates the strength in the range of $b=0.2-0.8$, as compared with the test results. Also noticed by Abelev and Lade (2004) and Lade $(2007,2008)$ is the occurrence of shear band before the peak strength state may reduce the strength measured from the boundary of the samples in this range. Shear banding may account for the observed discrepancy in Fig. 8.

\section{Conclusions}

A general and efficient approach has been proposed to develop an anisotropic failure criterion for soils with cross anisotropy. In essence, two variables relevant to fabric anisotropy were considered. One is the degree of cross anisotropy of a soil, and the other is the first joint invariant of the deviatoric stress tensor and the deviatoric fabric tensor, which physically represents the relative orientation between

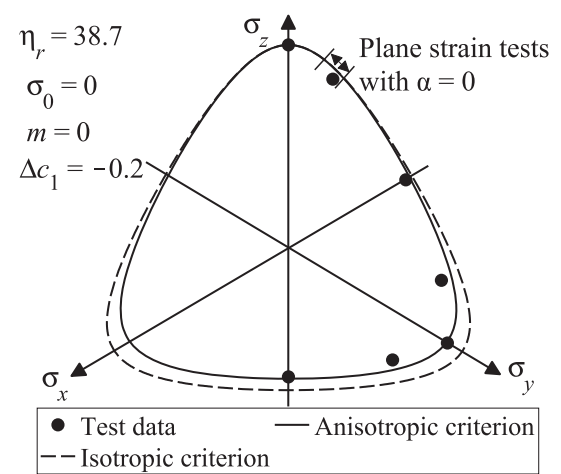

(a)

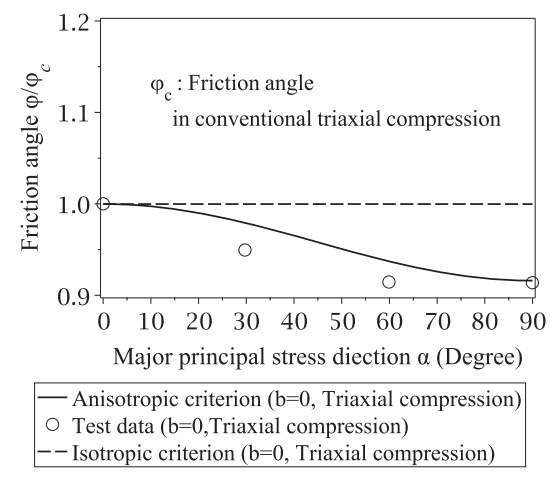

(b)

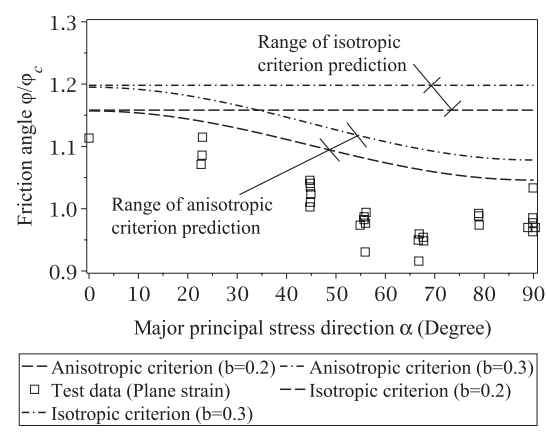

(c)

Fig. 7. Prediction of the failure of Toyoura sand by the isotropic and anisotropic criterion in (a) true triaxial tests; (b) triaxial compression tests; (c) plan strain tests [test data from Lam and Tatsuoka (1988) and Tatsuoka et al. (1990)]

stress direction and material fabric in the three-dimensional space. By modifying the parameters relevant to cohesion and frictional characteristics of an isotropic failure criterion to be a function of the anisotropic variable and the degree of anisotropy, the effect of fabric anisotropy may be properly taken into account. As an illustrative example, the well-received Lade's (1977) isotropic failure criterion has been employed to demonstrate the generalization procedure. The conventional triaxial compression shear mode where the major principal stress direction is perpendicular to the isotropic plane is used as a convenient reference state for calibration of the new criterion. The usefulness of the new failure criterion in characterizing the strength anisotropy in soils was validated with a wide range of sands and clays. The current general method was motivated by a previous attempt by the authors (Gao et al. 2010), which can be regarded as a specific implementation of the proposed approach in this paper. With a couple of more parameters involved, the underlying isotropic failure criterion used is also more complex 


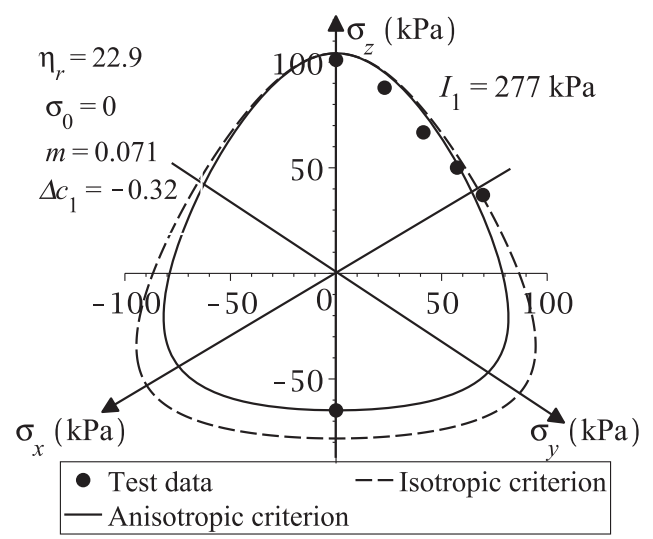

(a)

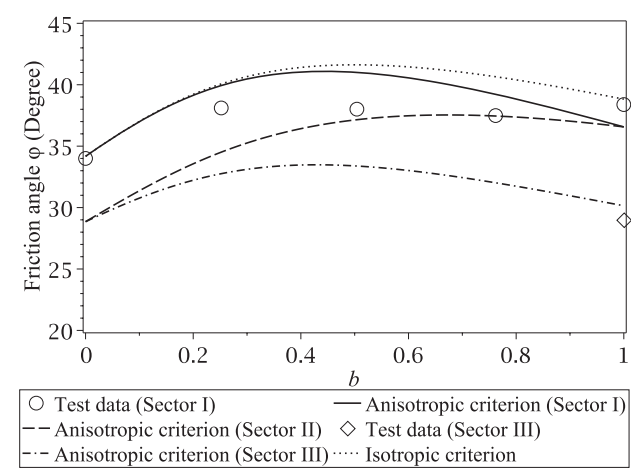

(b)

Fig. 8. Comparison between the true triaxial test data on Nevada II/ATC silty sand and predictions by the isotropic and anisotropic criteria in (a) the deviatoric plane; (b) the $b-\varphi$ plane [test data from Shapiro and Yamamuro (2003)]

than the Lade's criterion used. Also, the present generalized Lade criterion has also been applied to all the sands and clays discussed in Gao et al. (2010). Though not presented, the predictions were good when compared with the test data.

\section{Appendix. Calculation of the Anisotropic Variable $\boldsymbol{A}$}

\section{Calculation of A in Torsional Shear Tests}

For the representative element in the torsional shear tests shown in Fig. 1(a), the deviatoric fabric tensor can be expressed as

$$
d_{i j}=\left[\begin{array}{ccc}
d_{z} & 0 & 0 \\
0 & d_{\theta} & 0 \\
0 & 0 & d_{r}
\end{array}\right]=\frac{2 \Delta}{9+3 \Delta}\left[\begin{array}{rrr}
-2 & 0 & 0 \\
0 & 1 & 0 \\
0 & 0 & 1
\end{array}\right]
$$

The deviatoric stress components applied on the representative element is

$$
\begin{aligned}
s_{i j} & =\left[\begin{array}{ccc}
s_{z} & s_{z \theta} & 0 \\
s_{\theta z} & s_{\theta} & 0 \\
0 & 0 & s_{r}
\end{array}\right] \\
& =\frac{s_{3}}{1+b}\left[\begin{array}{ccc}
1+b-3 \cos ^{2} \alpha & -3 \sin \alpha \cos \alpha & 0 \\
-3 \sin \alpha \cos \alpha & 3 \cos ^{2} \alpha+b-2 & 0 \\
0 & 0 & 1-2 b
\end{array}\right]
\end{aligned}
$$

where $s_{3}(\leq 0)=$ minor deviatoric principal stress. According to the definition of $A$ expressed by Eq. (2), one can get Eq. (6) in conjunction with Eqs. (16) and (17). Eqs. (16) and (17) can also be used to calculate the expressions for $B, C$, and $D$ [Eqs. (3)-(5)]. Because the expressions are relatively complex, only the numerical results are shown in Figs. 1(c-e).

\section{Calculation of A True Triaxial Tests}

As for the true triaxial test condition shown in Fig. 2, the deviatoric fabric tensor for the sample is the same for all the three sectors

$$
\begin{aligned}
d_{i j} & =\left[\begin{array}{ccc}
d_{z} & 0 & d_{z y} \\
0 & d_{x} & 0 \\
d_{y z} & 0 & F_{y}
\end{array}\right] \\
& =\frac{2 \Delta}{9+3 \Delta}\left[\begin{array}{ccc}
1-3 \cos ^{2} \alpha & 0 & -3 \sin \alpha \cos \alpha \\
0 & 1 & 0 \\
-3 \sin \alpha \cos \alpha & 0 & 3 \cos ^{2} \alpha-2
\end{array}\right]
\end{aligned}
$$

and the applied deviatoric stress system can be expressed as the following:

1. Sector I

$$
s_{i j}=\left[\begin{array}{ccc}
s_{z} & 0 & 0 \\
0 & s_{x} & 0 \\
0 & 0 & s_{y}
\end{array}\right]=\frac{s_{3}}{1+b}\left[\begin{array}{ccc}
b-2 & 0 & 0 \\
0 & 1+b & 0 \\
0 & 0 & 1-2 b
\end{array}\right]
$$

2. Sector II

$$
s_{i j}=\left[\begin{array}{ccc}
s_{z} & 0 & 0 \\
0 & s_{x} & 0 \\
0 & 0 & s_{y}
\end{array}\right]=\frac{s_{3}}{1+b}\left[\begin{array}{ccc}
1-2 b & 0 & 0 \\
0 & 1+b & 0 \\
0 & 0 & b-2
\end{array}\right]
$$

3. Sector III

$$
s_{i j}=\left[\begin{array}{ccc}
s_{z} & 0 & 0 \\
0 & s_{x} & 0 \\
0 & 0 & s_{y}
\end{array}\right]=\frac{s_{3}}{1+b}\left[\begin{array}{ccc}
1+b & 0 & 0 \\
0 & 1-2 b & 0 \\
0 & 0 & b-2
\end{array}\right]
$$

According to Eqs. (2) and (18)- (21), the expression for $A$ in true triaxial tests shown in Fig. 2 can be obtained [Eq. (8)].

\section{Acknowledgments}

This work was supported by Research Grants Council of Hong Kong (under GRF 623211 and SBI08/09.EG02).

\section{References}

Abelev, A., and Lade, P. V. (2004). "Characterization of failure in crossanisotropic soils." J. Eng. Mech., 130(5), 599-606.

Anandarajah, A. M., and Dafalias, Y. F. (1986). "Bounding surface plasticity. III: Application to anisotropic cohesive soils." J. Eng. Mech., 112(12), 1292-1318. 
Azami, A., Pietruszczak, S., and Guo, P. (2010). "Bearing capacity of shallow foundations in transversely isotropic granular media." Int. J. Numer. Anal. Methods Geomech., 34(8), 771-793.

Brewer, R. (1964). Fabric and mineral analysis of soils, Wiley, New York.

Callisto, L., and Calabresi, G. (1998). "Mechanical behaviour of a natural soft clay." Geotechnique, 48(4), 495-513.

Callisto, L., and Rampello, S. (2002). "Shear strength and small-strain stiffness of a natural clay under general stress conditions." Geotechnique, 52(8), 547-560.

Casagrande, A., and Carillo, N. (1944). "Shear failure of anisotropic materials." J. Boston Soc. Civ. Eng., 31(4), 74-87.

Dafalias, Y. F., Papadimitriou, A. G., and Li, X. S. (2004). "Sand plasticity model accounting for inherent fabric anisotropy." J. Eng. Mech., 130(11), 1319-1333.

Gao, Z. W., Zhao, J. D., and Yao, Y. P. (2010). "A generalized anisotropic failure criterion for geomaterials." Int. J. Solids Struct., 47(22-23), $3166-3185$.

Guo, P., and Stolle, D. F. E. (2005). "On the failure of granular materials with fabric effects." Soil Found., 45(4), 1-12.

Haruyama, M. (1981). "Anisotropic deformation-strength properties of an assembly of spherical particles under three dimensional stresses." Soil Found., 21(4), 41-55.

Hill, R. (1950). Mathematical theory of plasticity, Oxford University Press, Oxford, U.K.

Kaliakin, V. N. (2003). "An assessment of the macroscopic quantification of anisotropy in cohesive soils." Proc., 1st Japan-U.S. Workshop Test. Model. Simulat., ASCE Geotechnical Special Publications GSP 143, Boston, 370-393.

Kumruzzaman, Md. (2008). "Experimental study on the stress-strainstrength behavior of a completely decomposed granite soil and a geofoam." Ph.D. thesis, Hong Kong Polytechnic Univ., Hong Kong.

Kumruzzaman, Md., and Yin, J.-H. (2010). "Influence of principal stress direction and intermediate principal stress on the stress-strain-strength behavior of completely decomposed granite." Can. Geotech. J., 47(2), 164-179.

Kurukulasuriya, L. C., Oda, M., and Kazama, H. (1999). "Anisotropy of undrained shear strength of an over-consolidated soil by triaxial and plane strain tests." Soil Found., 39(1), 21-29.

Lade, P. V. (1977). "Elasto-plastic stress-strain theory for cohesionless soil with curved yield surfaces." Int. J. Solids Struct., 13(11), 1019-1035.

Lade, P. V. (1997). "Modeling the strengths of engineering materials in three dimensions." Mech. Cohes.-Frict. Mater., 2(4), 339-356.

Lade, P. V. (2007). "Modeling failure in cross-anisotropic frictional materials." Int. J. Solids Struct., 44(16), 5146-5162.

Lade, P. V. (2008). "Failure criterion for cross-anisotropic soils." J. Geotech. Geoenviron. Eng., 134(1), 117-124.

Lade, P. V., Nam, J., and Hong, W. P. (2008). "Shear banding and crossanisotropic behavior observed in laboratory sand tests with stress rotation." Can. Geotech. J., 45(1), 74-84.

Lam, W. K., and Tatsuoka, F. (1988). "Effects of initial anisotropic fabric and $\sigma_{2}$ on strength and deformation characteristics of sand." Soil Found., 28(1), 89-106.

Li, X. S., and Dafalias, Y. F. (2002). "Constitutive modeling of inherently anisotropic sand behavior." J. Geotech. Geoenviron. Eng., 128(10), 868-880.

Liu, M. D., and Indraratna, B. N. (2011). "General strength criterion for geomaterials including anisotropic effect." Int. J. Geomech., 11(3), 251-262.

Matsuoka, H., and Nakai, T. (1974). "Stress-deformation and strength characteristics of soil under different principal stresses." Proc., Japan
Society of Civil Engineers (JSCE), Japan Society of Civil Engineers, Vol. 232, 59-70.

Mitchell, J. K., and Soga, K. (2005). Fundamentals of soil behavior, 3rd Ed., Wiley, Hoboken, NJ

Mortara, G. (2010). "A yield criterion for isotropic and cross-anisotropic cohesive-frictional materials." Int. J. Numer. Anal. Methods Geomech., 34(9), 953-977.

Muhunthan, B., Chameau, J. L., and Masad, E. (1996). "Fabric effects on the yield behavior of soils." Soil Found., 36(3), 85-97.

Nishimura, S., Minh, N. A., and Jardine, R. J. (2007). "Shear strength anisotropy of natural London clay." Geotechnique, 57(1), 49-62.

Ochiai, H., and Lade, P. V. (1983). "Three-dimensional behavior of sand with anisotropic fabric." J. Geotech. Eng., 109(10), 1313-1328.

Oda, M., Koishikawa, I., and Higuchi, T. (1978). "Experimental study of anisotropic shear strength of sand by plane strain test." Soils Found., 18 (1), 25-38.

Oda, M., and Nakayama, H. (1989). "Yield function for soil with anisotropic fabric." J. Eng. Mech., 115(1), 89-104.

O'Neill, D. A. (1985). "Undrained strength anisotropy of an overconsolidated thixotropic clay." M.S. thesis, Dept. of Civil Eng., MIT, Cambridge, MA.

Pietruszczak, S., and Mroz, Z. (2000). "Formulation of anisotropic failure criteria incorporating a microstructure tensor." Comput. Geotech., 26(2), 105-112.

Pietruszczak, S., and Mroz, Z. (2001). "On failure criteria for anisotropic cohesive-frictional materials." Int. J. Numer. Anal. Methods Geomech., 25(5), 509-524.

Schweiger, H. F., Wiltafsky, C., Scharinger, F., and Galavi, V. (2009). "A multilaminate framework for modelling induced and inherent anisotropy of soils." Geotechnique, 59(2), 87-101.

Sekiguchi, H., and Ohta, K. (1977). "Induced anisotropy and time dependency in clays." Proc., 9th Int. Conf. Soil Mech. Found. Eng., Japanese Society of Soil Mechanics and Foundation Engineering, Tokyo, 229-238.

Shapiro, S., and Yamamuro, J. A. (2003). "Effects of silt on threedimensional stress-strain behavior of loose sand." J. Geotech. Geoenviron. Eng., 129(1), 1-11.

Tatsuoka, F., Nakamura, S., Huang, C. C., and Tani, K. (1990). "Strength anisotropy and shear band direction in plane strain tests of sand." Soil Found., 30(1), 35-54.

Uthayakumar, M., and Vaid, Y. P. (1998). "Static liquefaction of sands under multiaxial loading." Can. Geotech. J., 35(2), 273-283.

Wang, C. C. (1970). "A new representation theorem for isotropic functions: An answer to Professor G. F. Smith's criticism of my papers on representations for isotropic functions." Arch. Ration. Mech. Anal., 36(3), 166-197.

Whittle, A. J., Degroot, D. J., Ladd, C. C., and Seah, T.-H. (1994). "Model prediction of anisotropic behavior of boston blue clay." J. Geotech. Eng., 120(1), 199-224.

Yao, Y. P., and Kong, Y. X. (2011). "Extended UH model: Threedimensional unified hardening model for anisotropic clays." J. Eng. Mech., 138(7), 853-866.

Yong, R. N., and Silvestri, V. (1979). "Anisotropic behaviour of a sensitive clay." Can. Geotech. J., 16(2), 335-350.

Yoshimine, M., Ishihara, K., and Vargas, W. (1998). "Effects of principal stress direction and intermediate principal stress on undrained shear behavior of sand." Soil Found., 38(3), 179-188.

Zdravković, L., Potts, D. M., and Hight, D. W. (2002). "The effect of strength anisotropy on the behavior of embankments on soft ground." Geotechnique, 52(6), 447-457. 